\section{All-optical wavelength conversion of nonreturn-to-zero signals based on a nonlinear polarization switch and a delayed interferometer}

\author{
Xiaofan Zhao, ${ }^{\text {a }}$ Caiyun Lou, ${ }^{a}$ and Shilong Pan ${ }^{b}$ \\ aTsinghua University, Department of Electronic Engineering, \\ TNList and State Key Laboratory on Integrated \\ Optoelectronics, Beijing 100084, China \\ E-mail: zhaoxf@mails.tsinghua.edu.cn \\ ${ }^{b}$ Nanjing University of Aeronautics and Astronautics, \\ College of Information Science and Technology, Nanjing, \\ Jiangsu 210016, China
}

\begin{abstract}
A novel all-optical wavelength converter for nonreturn-to-zero systems based on a nonlinear polarization switch (NPS) and a delayed interferometer (DI) is proposed and demonstrated experimentally. The performance of the proposed wavelength converter is compared with the performance of a conventional wavelength converter that only incorporates a NPS. Results show that the quality of the converted signal is significantly improved by inserting a DI after the NPS. The transmission performance of the wavelengthconverted signal is also improved by the DI. (C) 2010 Society of Photo-Optical Instrumentation Engineers. [DOI: 10.1117/1.3509381]
\end{abstract}

Subject terms: optical communication; semiconductor optical amplifier; wavelength converter; nonlinear polarization rotation; delayed interferometer.

Paper 100445LR received Jun. 2, 2010; revised manuscript received Oct. 6, 2010; accepted for publication Oct. 8, 2010; published online Nov. 19,2010

\section{Introduction}

The all-optical wavelength converter (WC) is a promising technology to overcome wavelength blocking and to perform switching functions in future scalable, low-latency, and transparent optical networks. WCs using nonlinear polarization switches (NPSs) based on the nonlinear polarization rotation effect in semiconductor optical amplifiers (SOAs) have attracted considerable research interest due to the advantages of their simple structure, selectable bit polarity, and high extinction ratio (ER). ${ }^{1-3}$ However, the quality of the converted signal is degraded by the pattern effect induced by the slow gain recovery time of the SOA. To overcome this problem, subsequent optical red-shifted filtering has been used to mitigate the pattern effect and accelerate the operating rate of the NPS. ${ }^{4,5}$ Delayed interferometers (DIs) have also been used to improve the performance of SOA-based WCs. ${ }^{6-8}$ Since the DI is considered to be effective when narrow input pulses are used, it is mainly applied in return-to-zero (RZ) systems. Although WCs based on NPS and DI have been demon-

0091-3286/2010/\$25.00 @ 2010 SPIE strated respectively, little attention has been paid on the possible contributions from DI to improving the performance of SOA-based WC for nonreturn-to-zero (NRZ) systems. ${ }^{9}$ In this work, a novel WC for NRZ systems is proposed and demonstrated using a SOA-based NPS and a DI. The high quality of the converted signal out of the NPS-DI WC is confirmed by the negative power penalty after transmission over a $25-\mathrm{km}$ standard single-mode fiber (SMF). The proposed system is also applicable to multicasting operations.

\section{Experimental Demonstration}

Figure 1 shows the experimental setup of the proposed WC based on the NPS-DI configuration. A continuous-wave (CW) light at $1543.86 \mathrm{~nm}$ is generated by a distributed feedback (DFB) laser diode and modulated at a Mach-Zehnder modulator by a $10-\mathrm{Gb} / \mathrm{s} \mathrm{NRZ} \mathrm{pseudorandom} \mathrm{bit} \mathrm{sequence}$ (PRBS) with a word length of $2^{31}-1$. The generated NRZ signal and another $\mathrm{CW}$ probe light at $1549.44 \mathrm{~nm}$ are injected to a SOA (SOA-NL-OEC-1550 from CIP, Ipswich, United Kingdom) via a 3-dB coupler. The bias current of the SOA is $250 \mathrm{~mA}$. The input power of the $\mathrm{CW}$ probe and the data signal is -0.8 and $4.8 \mathrm{dBm}$, respectively. The proposed WC consists of a conventional SOA-based NPS and a DI. Two polarization controllers (PCs) combined with a polarizer are used to achieve the NPS function, which is based on the birefringence effect in the SOA. The birefringence would be further enhanced by carefully adjusting the polarization direction of the injected data signal. The transverse electric (TE) and transverse magnetic (TM) components of the probe light will obtain different gains and nonlinear phase shift due to the asymmetry between the two modes. This process is equivalent to polarization modulation of the probe light by the intensity of the data signal. The first PC is used to adjust the polarization of the probe light to have an angle of $45 \mathrm{deg}$ to the principal axes of the SOA, which maximizes polarization modulation. The second PC is used to align the polarization direction of the amplified probe light to be orthogonal to the principal axis of the polarizer, which converts the polarization modulation in the probe light into intensity modulation at the polarizer. An optical bandpass filter with a $3-\mathrm{dB}$ bandwidth of $1 \mathrm{~nm}$ is inserted at the output of the SOA to block the data signal and suppress the amplified spontaneous emission (ASE) generated by the SOA. To improve the signal quality, we incorporate a DI at the output of the NPS. The DI used in the experiment consists of two PCs, a polarizer, and a variable differential group delay (DGD) element. The delay between the two arms of the DI is set to be $30 \mathrm{ps}$. To examine the signal quality, the converted signals are transmitted through 80-km dispersion-managed fiber link composed of 80-km SMF and a dispersion compensation module [Corning (Corning, New York) PureForm DCM SC-B-080-L-SCUPC-150-01]. The transmission loss of the fiber link is compensated for by an erbium-doped optical fiber amplifier (EDFA) placed after the fiber link. The amplified signal is filtered by another 1-nm optical bandpass filter and sent into a tunable optical attenuator before entering the receiver. The optical signals are detected by a $50-\mathrm{GHz}$ photodiode and observed by a sampling oscilloscope [Agilent (Santa Clara, California) 86100A]. The bit error rate (BER) curves are measured by a BER tester (Agilent, N4901B).

The operation principles of the NPS-DI scheme are as follows. First, a noninverted wavelength-converted signal based 


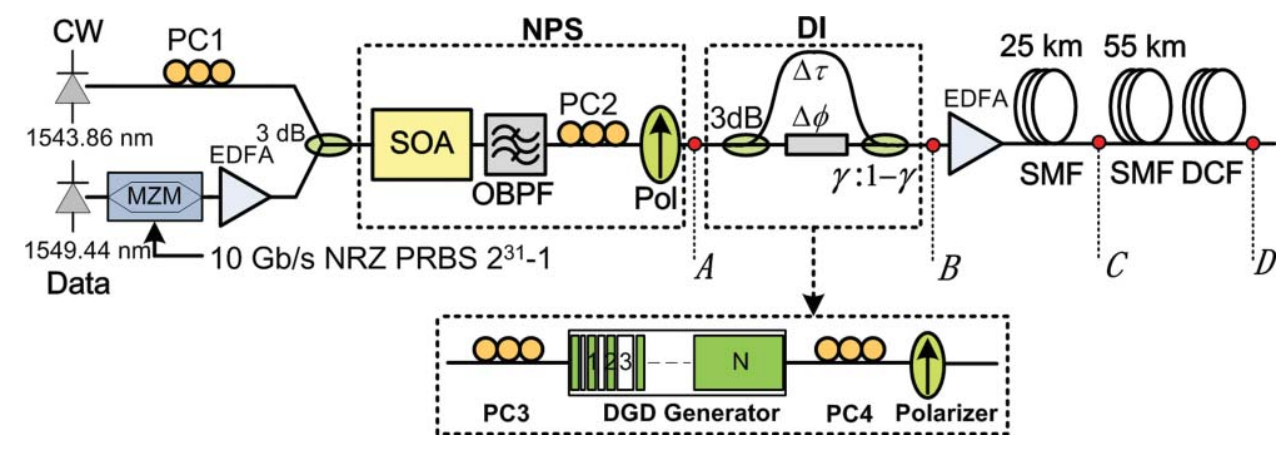

Fig. 1 Experimental Setup.

on the conventional NPS is obtained at the output of the polarizer. A typical waveform of the noninverted signal measured at point $\mathrm{A}$ is shown in Fig. 2. The converted signal presents slow rising and falling edges that are mainly induced by the limited response of the SOA. Figure 2 shows a time-resolved chirp of the noninverted wavelength converted signal measured using an optical bandpass filter. ${ }^{10}$ It is well known that cross-phase modulation (XPM) accompanies the NPR in the SOA. The XPM effect would impose a red-shift chirp on the leading edge and a blue-shift chirp on the trailing edge for noninverted wavelength conversion. As can be seen, the leading and trailing edges of the converted signal are oppositely chirped, which means that the signal quality can be improved by the technique of subsequent spectral filtering. ${ }^{11}$ If the DI is used as a notch filter and properly biased to suppress the blue-shifted falling edge and enhance the red-shifted rising edge of the converted signal, the waveform would be reshaped to restore the high signal quality. Besides, since the central wavelength of the converted signal is not located at the minimum transmission point of the DI, the cancelling of the consecutive 1's in the NRZ signal is avoided.

The eye diagrams of the input and converted signals are shown in the insets in Fig. 3. The input signal has an ER of $13.9 \mathrm{~dB}$ and a Q-factor of 18.2, respectively. After the NPS, the signal quality is degraded by the pattern effect, which is induced by the slow gain recovery time of the SOA. The eye diagram of the converted signal presents slow rising and falling edges. The ER and Q-factor of the converted signal is only $10.6 \mathrm{~dB}$ and 6.2, respectively, which results in a power penalty of $4 \mathrm{~dB}$ at a BER of $10^{-9}$. Figure 4 shows the optical spectra of wavelength converted signals before and after DI. The transmission of the DI is also measured by using a polarized ASE source generated by blocking the input lights to the SOA. As shown in Fig. 4, the DI is adjusted to locate the carrier of the NPS-based converted signal on the positive slope of the DI transmission spectrum in the wavelength domain. Thus, the red-chirped rising edge of the NPS converted signal will receive larger transmittance than the blue-chirped falling edge due to the detuned filtering effect of the DI. Suppression of the blue-shifted frequency component and enhancement of the red-shifted frequency component are simultaneously realized, and therefore the rising and falling time of the converted signal is reduced due to the mitigation of the pattern effects. The eye diagram of the signal is more opened than before the DI. The ER and Q-factor are greatly improved and increased to $12.2 \mathrm{~dB}$ and 9.2 , respectively. Correspondingly, the power penalty of the wavelength conversion is reduced by $1.5 \mathrm{~dB}$. The Q-factor

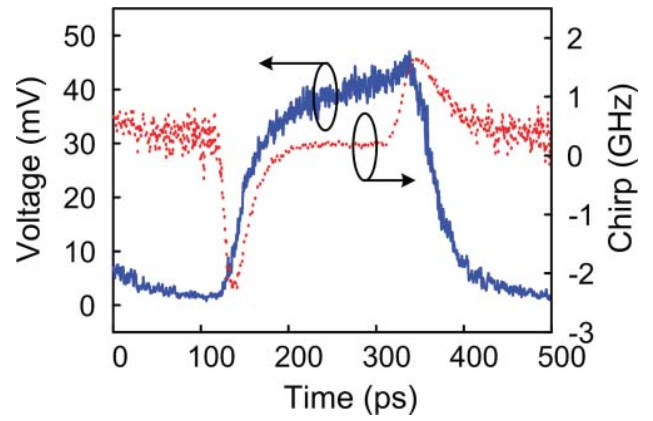

Fig. 2 Measured waveform (solid line) and time-resolved chirp (dotted line) of the noninverted converted signal by NPS with an input data signal of 0110 .

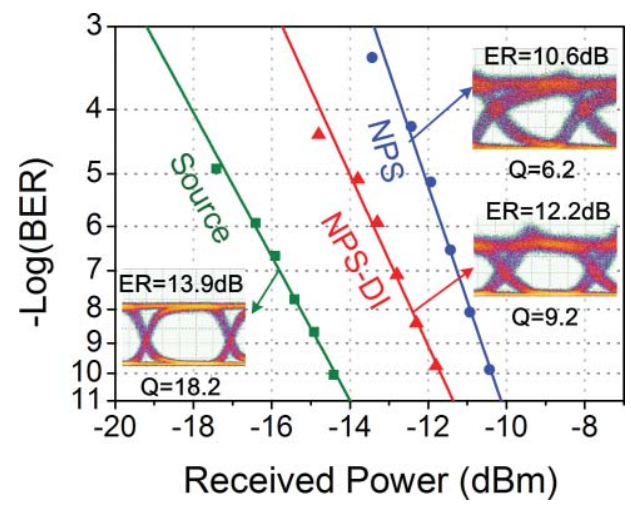

Fig. 3 BER curves and eye diagrams of the back-to-back signal and the wavelength converted signals using NPS and NPS-DI.

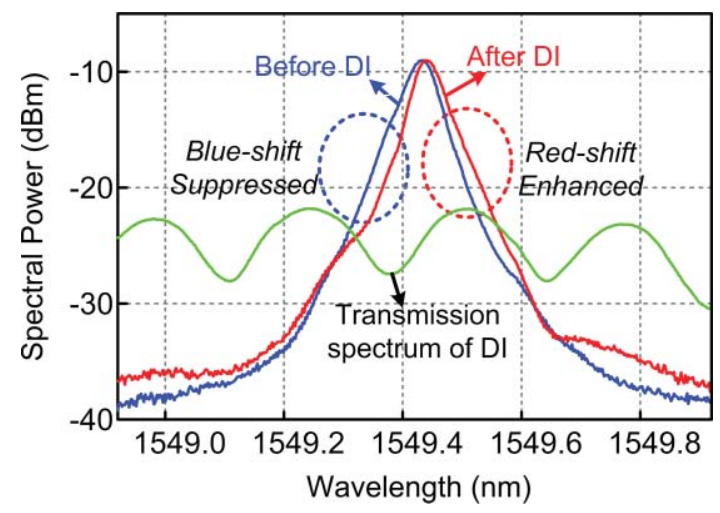

Fig. 4 Measured spectra of the converted signal before and after DI, and the transmission spectrum of the DI. 


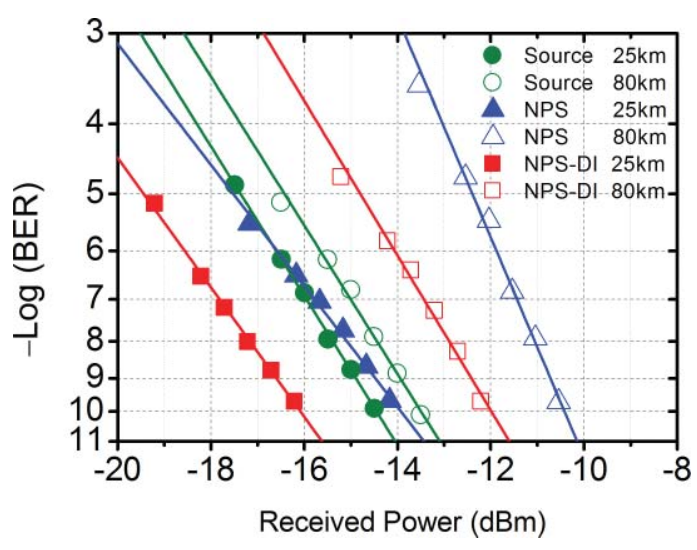

Fig. 5 BER results of the source and the converted signals at the output of the NPS and of the NPS-DI after transmission.

and the BER performance of the converted signal indicate that the ASE noise generated by the SOA would not degrade the signal quality severely. Furthermore, it can be seen from Fig. 4 that the DI is actually a multichannel filter with the free spectral range in inverse proportion to the delay between the two arms. Given that NPS itself supports multicasting operation, it is possible to obtain multicasting operation with enhanced performance of all the channels using the proposed NPS-DI WC.

The transmission performance of the converted signals is also investigated to evaluate the signal quality. The eye diagrams and the BER results of the source and converted signals are shown in Fig. 5. The receiver sensitivity of the source after transmission over 25-km SMF without dispersion compensation is improved due to the small chirp of the modulator. The converted signal out of NPS-DI has a positive chirp across the converted signal, which is opposite to that of the SMF. As a result, the converted NRZ signal is compressed and evolves into a RZ-like waveform after transmission through $25 \mathrm{~km}$ of SMF, leading to improvement of the receiver sensitivity. In addition, the suppression of the pattern effects also enhances the transmission performance of the NPS-based converted signal, and therefore a negative power penalty of $-1.5 \mathrm{~dB}$ is obtained. For the transmission over 80-km dispersion-managed fiber link, the power penalty of the NPS-based converted signal is $3.2 \mathrm{~dB}$, and is improved to be $1.5 \mathrm{~dB}$ for the converted signal out of the DI due to the signal reshaping by the detuned filtering of the DI.

\section{Conclusions}

We propose and demonstrate a novel all-optical WC for NRZ systems based on the NPS-DI scheme. Due to pulse reshaping in the DI, the Q-factor and ER of the NPS-based converted signal are greatly improved. The receiver sensitivity of the NPS-based converted signal at a BER of $10^{-9}$ after transmission over $25 \mathrm{~km}$ of SMF and $80 \mathrm{~km}$ of dispersion-managed fiber link is improved by 2 and $1.5 \mathrm{~dB}$, respectively, indicating that the performance of the NPS-based wavelength converter can be improved by the detuned filtering of a following DI.

\section{Acknowledgment}

This work was supported by project 60736036 supported by NSFC, the project 2009AA01Z256 supported by 863, and the project YB20091000301 by Beijing.

\section{References}

1. H. Soto, D. Erasme, and G. Guekos, "Cross-polarization modulation in semiconductor optical amplifiers," IEEE Photon. Technol. Lett. 11 970-972 (1999)

2. Y. Liu, M. T. Hill, E. Tangdiongga, H. d. Waardt, N. Calabretta, G. D. Khoe, and H. J. S. Dorren,"Wavelength conversion using nonlinear polarization rotation in a single semiconductor optical amplifier," Photon. Technol. Lett., IEEE 15, 90-92 (2003).

3. G. Contestabile, N. Calabretta, M. Presi, and E. Ciaramella, "Single and multicast wavelength conversion at $40 \mathrm{~Gb} / \mathrm{s}$ by means of fast nonlinear polarization switching in an SOA," Photon. Technol. Lett. IEEE 17, 2652-2654 (2005).

4. J. Zhang, J. Wu, K. Xu, and J. Lin, "All-optical wavelength conversion based on nonlinear polarization rotation (NPR) in SOA and AWG filtering," Chin. Opt. Lett., 4, 205-207 (2006).

5. J. Zhang, J. Wu, C. Feng, K. Xu, and J. Lin, "All-optical logic gate exploiting nonlinear polarization rotation in an SOA and red-shifted sideband filtering," IEEE Photon. Technol. Lett., 19(1), 33-35 (2007).

6. Y. Ueno, S. Nakamura, K. Tajima, and S. Kitamura, "3.8-THz wavelength conversion of picosecond pulses using a semiconductor delayed-interference signal-wavelength converter (DISC),' IEEE Photon. Technol. Lett., 10(3) 346-348 (1998).

7. J. Leuthold, C. H. Joyner, B. Mikkelsen, G. Raybon, J. L. Pleumeekers, B. I. Miller, K. Dreyer, and C. A. Burrus, "100 Gbit/s all-optical wavelength conversion with integrated SOA delayed-interference configuration," Electron. Lett. 36(17), 1129-1130 (2000).

8. N. Y. Kim, X. Tang, J. C. Cartledge, and A. K. Atieh, "Design and performance of an all-optical wavelength converter based on a semiconductor optical amplifier and delay interferometer," J. Lightwave Technol. 25. 3730-3738 (2007).

9. W. Chia Chien, H. Ming Fang, and J. Chen, "Enhancing the frequency response of cross-polarization wavelength conversion," IEEE Photon. Technol. Lett. 17, 1683-1685 (2005).

10. M. Matsuura, N. Iwatsu, K. Kitamura, and N. Kishi, "Time-resolved chirp properties of SOAs measured with an optical bandpass filter," IEEE Photon. Technol. Lett., 20(23), 2001-2003 (2008).

11. M. L. Nielsen and J. Mørk, "Increasing the modulation bandwidth of semiconductor-optical-amplifier-based switches by using optical filtering," J. Opt. Soc. Am. B. 21, 1606-1619 (2004). 